\title{
Clinical Outcomes of Endovascular Treatment within 24 Hours in Patients with Mild Ischemic Stroke and Perfusion Imaging Selection
}

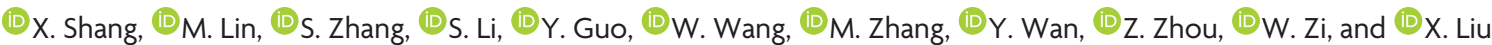

\begin{abstract}
BACKGROUND AND PURPOSE: Endovascular thrombectomy has been accepted as the standard of care for patients with acute ischemic stroke. Our aim was to investigate the clinical outcomes of patients with mild ischemic stroke with acute proximal large-vessel occlusion after endovascular treatment within 24 hours of symptom onset.
\end{abstract}

MATERIALS AND METHODS: Between January 2014 and August 2017, ninety-three Chinese patients with mild ischemic stroke (NIHSS scores, 0 -8) and large-vessel occlusion with endovascular treatment were retrospectively enrolled from 7 comprehensive stroke centers. They were divided into 2 groups: $\leq 6$ hours and 6-24 hours from symptom onset to groin puncture. We analyzed their modified Rankin Scale scores at 90 days, symptomatic intracranial hemorrhage at 48 hours, and mortality during 90 days. Multivariable linear regression analysis was used to identify predictors for NIHSS shift after discharge.

RESULTS: Twenty-nine patients received endovascular treatment within 6-24 hours after symptom onset and had an imaging mismatch based on perfusion CT or diffusion-weighted MR imaging. There were no substantial differences between the 2 groups in 90 -day functional independence $(P=.54)$ and the risks of the combination of symptomatic intracranial hemorrhage and death $(P=.72)$. Two significant indicators of NIHSS shift were 48-hour symptomatic intracranial hemorrhage (unstandardized $\beta=7.28 ; 95 \% \mathrm{Cl}, 3.48-11.1 ; P<.001$ ) and baseline systolic blood pressure (unstandardized $\beta=0.08 ; 95 \% \mathrm{Cl}, 0.03-0.14 ; P=.005$ ).

CONCLUSIONS: Patients with mild ischemic stroke and large-vessel occlusion in the anterior circulation, an imaging mismatch, and endovascular treatment within 6-24 hours of initial symptoms showed no heterogeneity in the efficacy and safety outcome compared with those treated $\leq 6$ hours from symptom onset.

ABBREVIATIONS: LVO = large-vessel occlusion; $\mathrm{MIS}=$ mild ischemic stroke; $\mathrm{sICH}$ = symptomatic intracranial hemorrhage

E ndovascular thrombectomy has been accepted as the standard of care for patients with acute anterior large-vessel occlusion (LVO) since the publication of 5 randomized clinical trials in

Received January 4, 2018; accepted after revision February 26.

From the Department of Neurology (X.S., S.Z., Y.G., X.L.), Jinling Clinical College of Nanjing Medical University, Nanjing, Jiangsu, China; Department of Neurology (X.S., Z.Z.), Yijishan Hospital of Wannan Medical College, Wuhu, Anhui, China; Department of Neurology (M.L.), Fuzhou General Hospital of Nanjing Military Region, Fuzhou, Fujian, China; Department of Neurology (S.Z.), Affiliated Hospital of Yangzhou University, Yangzhou University, Yangzhou, Jiangsu, China; Department of Neurology (S.L., W.Z., X.L.), Jinling Hospital, Southern Medical University, Nanjing, Jiangsu, China; Department of Neurology (Y.G.), Huai'an First People's Hospital, Nanjing Medical University, Huai'an, Jiangsu, China; Department of Radiology (W.W.), First People's Hospital of Yangzhou, Yangzhou University, Yangzhou, Jiangsu, China; Department of Neurology (M.Z.), Research Institute of Surgery, Daping Hospital, Third Military Medical University, Chongqing, China; and Department of Neurology (Y.W.), Hubei Zhongshan Hospital, Wuhan, Hubei, China.

This study was partly supported by the National Natural Science Foundation of China (No. 81530038, 81400993 and 81671172), the National Key Research and Development Program (No.2017YFC1307901), the Chinese Postdoctoral Science Fund (No. 2015M572815), and the scientific research fund of Wannan Medical College in Anhui Province, China to X. Shang (WK2016F32, Wuhu).

Xianjin Shang and Min Lin contributed equally to this work.
2015. ${ }^{1-5}$ Now, screening eligible patients and allowing the treatment to benefit more patients, including those with mild and progressive stroke, wake-up stroke, and unknown onset of ischemic events, are of great interest.

Patients with mild ischemic stroke (MIS) account for $>15 \%$ of patients with acute ischemic stroke. ${ }^{6}$ MIS presents with slight clinical deficit, rapidly improving symptoms, and an uncertain aggressive therapy risk-benefit ratio, which often confuses the clinician's decision. ${ }^{7}$ Due to the fragile collateral circulation in patients with MIS and LVO, their clinical symptoms could worsen gradually in the short term, and they may finally have severe dis-

Please address correspondence to Xinfeng Liu, MD, PhD, Department of Neurology, Jinling Hospital, Jinling Clinical College of Nanjing Medical University, Nanjing 210002, Jiangsu, China; e-mail: xfliu2@vip.163.com, and Wenjie Zi, MD, PhD, Department of Neurology, Jinling Hospital, Jinling Clinical College of Nanjing Medical University, Nanjing 210002, Jiangsu, China; e-mail: ziwenjie1981@163.com

- Indicates open access to non-subscribers at www.ajnr.org

三 Indicates article with supplemental on-line table.

http://dx.doi.org/10.3174/ajnr.A5644 
ability, so timely endovascular recanalization remains critical. ${ }^{8-11}$ However, further studies of high quality are needed to confirm the efficacy and safety of thrombectomy for these patients.

Recently, the DWI or CTP Assessment with Clinical Mismatch in the Triage of Wake Up and Late Presenting Strokes Undergoing Neurointervention with Trevo (DAWN) study confirmed that patients with acute ischemic stroke (NIHSS scores, $\geq 10$ ) with a mismatch between the severity of the clinical deficit and the infarct volume and undergoing thrombectomy within 6-24 hours have similar efficacy and safety profiles compared with those treated within 6 hours of symptom onset. ${ }^{12}$ However, whether this finding applies to patients with MIS (NIHSS scores, $\leq 8$ ) treated $>6$ hours after the onset of symptoms is unknown. The purpose of this study was to retrospectively evaluate the efficacy and safety of endovascular treatment performed in patients with acute stroke with NIHSS scores of $0-8$ within 24 hours of symptom onset.

\section{MATERIALS AND METHODS \\ Patients}

We retrospectively enrolled consecutive patients with ischemic stroke in China who were admitted to 7 comprehensive stroke centers (Jinling Hospital, Yijishan Hospital, Fuzhou General Hospital of Nanjing Military Region, Affiliated Hospital of Yangzhou University, Daping Hospital, Hubei Zhongshan Hospital, and No.123 Hospital of the People's Liberation Army) between January 2014 and August 2017 within 24 hours of symptom onset and who had LVO in the anterior circulation (MCA M1/M2, ICA, anterior cerebral artery) but not arterial aneurysms and arteriovenous malformations by CTA/MRA.

All eligible patients met the following inclusion criteria: 1) 18 years of age or older; 2) presenting with mild neurologic deficits with an admission NIHSS score of $\leq 8$; and 3) emergency endovascular treatment within 24 hours, including primary treatment and rescue treatment. Patients without prior functional independence ( $m R S$ score of $>2$ ) and those with intra-arterial thrombolysis alone were excluded.

Most patients received endovascular treatment with $\leq 6$ hours from symptom onset (group A). Patients who received endovascular recanalization therapy $>6$ hours after symptom onset, whose clinical presentations were disproportionately severe relative to the occlusive artery verified by CTA/MRA, and who had an imaging mismatch by perfusion CT or diffusion-weighted MR imaging were assigned to group $\mathrm{B}$.

This study was approved by the ethics committee of each participating center, but written consent was waived due to its retrospective nature.

\section{Data}

We retrieved the demographic, clinical, and neuroimaging data from each center. We included various data: age, sex, vascular risk factors (hypertension, hyperlipidemia, diabetes, smoking, and drinking habits), medical history (coronary heart disease, atrial fibrillation, history of stroke or TIA), baseline blood glucose level, systolic blood pressure and total cholesterol level, intravenous thrombolysis, occlusion sites, stroke etiology, time from onset to treatment, time from puncture to reperfusion, NIHSS scores (base- line, 24 hours, and discharge), ASPECTS, collateral flow scores, modified TICI score, symptomatic intracranial hemorrhage (sICH) at 48 hours, mortality, and $\mathrm{mRS}$ scores during 90 days.

All radiologic data and clinical medical records of subjects were sent to the core laboratory in our hospital (Jinling Hospital) and were reviewed in a blinded fashion by 2 physicians or interventionists (Y.G. and S.Z.) with advice of a third experienced physician/interventionist (W.Z.) when there was disagreement.

\section{Definition}

The etiology of stroke was divided into 3 categories (large atherosclerosis, cardioembolism, and others/undetermined) based on the Trial of ORG 10172 in Acute Stroke Treatment classification. ${ }^{13}$ Neurologic deterioration at 24 hours was defined as a $\geq 4$-point increase from the baseline NIHSS score. NIHSS shift represented the discharge NIHSS score minus the baseline NIHSS score.

The occlusion site of the vessel was divided into ICA, MCA M1, MCA M2, anterior cerebral artery, and tandem occlusion corresponding to the artery occlusion occurring in $>2$ different parts of continuous vessels. The collateral flow grading system was used to evaluate collateral flow, ranging from grade 0 to 4 , with zero representing no collateral flow, and 4 , rapid and complete collateral flow. ${ }^{14}$ The reperfusion status after the procedure was regarded as the modified TICI scale: $0-2 \mathrm{a}$ and $2 \mathrm{~b}-3$, which were divided by reperfusion of $50 \%$ of the affected vascular territory. ${ }^{15} \mathrm{sICH}$ at 48 hours after admission was any hemorrhage combined with an increase of $\geq 4$ points in the total NIHSS score or $\geq 2$ points in 1 NIHSS category according to the Heidelberg classification scheme. ${ }^{16}$

\section{Clinical and Safety Outcomes}

The primary outcome included functional outcome at 90 days, neurologic deterioration at 24 hours, and NIHSS shift at discharge. The functional outcomes were evaluated with the mRS scale at 90 days after symptom onset, assigned a score of $0-1,0-2$, and 3-6, respectively, representing excellent outcome, functional independence, and poor outcome. Patients' mRS scores at 3 months were retrieved from the stroke registry data base of each center, available either by telephone follow-up or outpatient visit. If patients were lost to follow-up, the 90-day mRS was replaced by the discharge mRS. ${ }^{17}$ Secondary safety outcomes included the incidence of sICH at 48 hours and mortality during 90 days.

\section{Statistical Analysis}

Quantitative variables are shown as mean \pm SD or median (interquartile range) as appropriate. Qualitative variables are presented as number and percentage. Comparisons between the 2 groups for quantitative/ordinal variables were made with the Student $t$ test, Mann-Whitney $U$ test, or analysis of variance, as appropriate. Qualitative variables were compared by the Pearson $\chi^{2}$ or Fisher exact test, as appropriate. Significance was set at $P<.05$, and $P$ values were 2-sided. Multivariable linear regression analysis for predictors of NIHSS shift was performed for variables at the .01 level of significance on univariate analysis (enter selection method). Statistical analysis was performed using SPSS Statistics 24.0 (IBM, Armonk, New York). 


\begin{tabular}{|c|c|c|c|c|c|c|c|c|}
\hline \multirow[b]{2}{*}{ Outcomes } & \multicolumn{4}{|c|}{ Endovascular Treatment } & \multicolumn{4}{|c|}{ Mechanical Thrombectomy } \\
\hline & All & $\begin{array}{c}\text { Group A } \\
(n=64)\end{array}$ & $\begin{array}{l}\text { Group B } \\
(n=29)\end{array}$ & $\begin{array}{c}P \\
(A \text { vs } B)\end{array}$ & All & $\begin{array}{c}\text { Group A } \\
(n=56)\end{array}$ & $\begin{array}{c}\text { Group B } \\
(n=23)\end{array}$ & $\begin{array}{c}P \\
(A \text { vs } B)\end{array}$ \\
\hline \multicolumn{9}{|l|}{ NIHSS score } \\
\hline Deterioration at $24 \mathrm{hr}$ & $13(14.0)$ & $7(10.9)$ & $6(20.7)$ & .22 & 12 (15.2) & $6(10.7)$ & $6(26.1)$ & .09 \\
\hline NIHSS shift at discharge ${ }^{b}$ & -0.6 & -0.6 & -0.5 & .94 & -0.4 & -0.6 & 0.1 & .73 \\
\hline \multicolumn{9}{|l|}{$\mathrm{mRS}$ at 90 days } \\
\hline mRS 0-1 & $55(59.1)$ & $41(64.1)$ & $14(48.2)$ & .15 & $46(58.2)$ & $35(62.5)$ & $11(47.8)$ & .23 \\
\hline mRS 0-2 & $68(73.1)$ & $48(75.0)$ & $20(69.0)$ & .54 & $56(70.9)$ & $42(75.0)$ & $14(60.9)$ & .20 \\
\hline \multicolumn{9}{|l|}{ Safety outcome } \\
\hline sICH at $48 \mathrm{hr}$ & $8(8.6)$ & $6(9.4)$ & $2(6.9)$ & 1.00 & $8(10.1)$ & $6(10.7)$ & $2(8.7)$ & 1.00 \\
\hline 90-Day mortality & $5(5.4)$ & $4(6.3)$ & $1(3.4)$ & 1.00 & $4(5.1)$ & $3(5.4)$ & $1(4.3)$ & 1.00 \\
\hline Composite outcome & $10(10.8)$ & $8(12.5)$ & $2(6.9)$ & .72 & 9 (11.4) & $7(12.5)$ & $2(8.7)$ & 1.00 \\
\hline
\end{tabular}

${ }^{a}$ Results are shown as number (\%) unless stated otherwise.

${ }^{b}$ Excluding death.

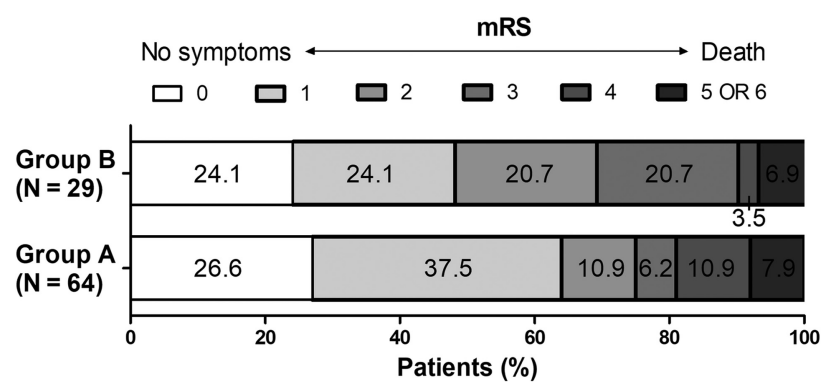

FIGURE. Distribution of $\mathrm{mRS}$ at 90 days between group $\mathrm{A}$ (onset to treatment $\leq 6$ hours) and group B (onset to treatment $>6$ hours).

\section{RESULTS}

\section{Patients}

A total of 93 patients met the inclusion criteria during the study period. The mean age of eligible patients was $61.6 \pm 13.8$ years, and $68.8 \%(64 / 93)$ of patients were men (On-line Table). The ASPECTS median was 9 (interquartile range, 8-10; except for the missing data); $30.1 \%$ (28/93) of patients had poor collateral status before the procedure; the overall proportion of intravenous thrombolysis was $28 \%$; and after endovascular treatment, $>90 \%(84 / 93)$ of patients had restored distal reperfusion (modified TICI 2b-3).

There were $64(68.8 \%)$ patients who received endovascular treatment within 6 hours after onset (group A), more than the 29 $(31.2 \%)$ patients who received treatment $>6$ hours after symptom onset (group B). The proportion of intravenous thrombolysis between the 2 groups was similar (28.1\% versus $27.6 \%, P=$ $.96)$, and the times from onset to intravenous thrombolysis were similar (130.0 \pm 72.7 minutes versus $141.6 \pm 107.7$ minutes; $P=$ .76). Other baseline characteristics were comparable across the 2 groups, though there were slight differences in stroke etiology composition and hypertension (both $P<.05$ ).

\section{Clinical Outcomes}

As shown in the Table and the Figure, the excellent outcome (mRS $0-1)$ in patients with endovascular treatment was higher in group A compared with group B (64.1\% versus $48.2 \%, P=.15)$, and the percentages of functional independence (mRS 0-2) were similar (75\% versus $69 \%, P=.54$ ). Of the 79 patients in the subgroup undergoing thrombectomy, the overall functional independence and excellent outcome were $70.9 \%$ and $58.2 \%$, respectively, and good outcomes at 90 days were more common in the group treated within 6 hours, but these patients did not show any substantial differences compared with those in the group treated beyond 6 hours (Table).

The shift in NIHSS scores indicated that both groups had slight improvement in symptoms at discharge $(-0.6$ versus -0.5 , $P=$.94). Multivariable linear regression of screening predictors for a shift in NIHSS scores indicated that sICH at 48 hours (unstandardized $\beta=7.28 ; 95 \%$ CI, 3.48-11.1; $P<.001$ ) and baseline systolic blood pressure (unstandardized $\beta=0.08$; 95\% CI, $0.03-$ $0.14 ; P=.005$ ) were associated with a harmful (positive) NIHSS shift. Intravenous thrombolysis, stroke etiology and occlusion site, reperfusion status, and other medical histories did not reach statistical significance in the multivariable analysis.

\section{Safety Outcomes}

sICH at 48 hours was not substantially different between the 2 endovascular groups (group A, 6 [9.4\%]; group B, 2 [6.9\%]). There were $4(6.3 \%)$ deaths within 3 months in group A, 1 after discharge and 3 during hospitalization: One case of postprocedural symptomatic hemorrhage was associated with death from a severe cerebral hernia, 1 case of a surgical complication resulted in rupture of a blood vessel, and the remaining patient died of revascularization failure. The only fatal case $(3.4 \%)$ in group B involved intracranial hemorrhage due to a procedural complication, and emergency removal of the hematoma was unable to save the patient.

\section{DISCUSSION}

Our study shows that in patients with acute ischemic stroke with LVO in the anterior circulation presenting with mild neurologic deficits inconsistent with occlusion site and imaging mismatch, the clinical and safety outcomes of endovascular therapy $\leq 6$ hours and 6-24 hours after symptom onset are not substantially different. Baseline systolic blood pressure and 48-hour sICH were the main factors influencing the neurologic recovery at discharge.

Previous studies have shown that patients with mild stroke with LVO tend to have poor outcomes if not treated. ${ }^{7,8}$ Mokin et $\mathrm{al}^{18}$ studied 204 patients with mild acute stroke (NIHSS scores, $0-7$ ) with LVO who were excluded from intravenous thrombolysis and endovascular treatment and found that $37 \%$ could not walk independently at discharge. Additionally, they found that 
the higher the admission score, the worse the clinical outcome was. ${ }^{18}$ In this study, we found that the overall functional independence of patients with endovascular therapy was $73.1 \%$ at 3 months, with 75\% for group A and 69\% for group B, respectively. Among the mechanical thrombectomy subgroup, the 90-day functional independence rate $(70.9 \%)$ was comparable with the findings of Dargazanli et $\mathrm{al}^{19}(78.3 \%)$ in patients with NIHSS scores in the range of $0-7$; the findings of Pfaff et $\mathrm{al}^{20}(63.6 \%)$ in patients with NIHSS scores of $\leq 8$; and the findings of Dargazanli et $\mathrm{al}^{21}$ and the ETIS Registry investigators in patients with NIHSS scores of $<8$ (81.2\%). Of note, in our patients with 6-24 hours from symptom onset to treatment, salvageable brain tissue was confirmed by perfusion CT or MR imaging mismatch and mismatched neurologic deficits inconsistent with the occlusion vessels. These patients undergoing mechanical thrombectomy still presented with favorable clinical outcomes, having comparable functional independence even within a prolonged time window (60.9\%), compared with patients treated within 6 hours from the present study (75\%) and above studies. ${ }^{19,21}$

The safety outcomes in our study were acceptable, with the 48-hour sICH and 90-day mortality being $8.6 \%$ and $5.4 \%$, respectively. These outcomes compare with 19.6\% (any ICH) and 5.1\% reported by Dargazanli et al, ${ }^{19}$ the $6.1 \%$ and $9.1 \%$ reported by Pfaff et al, ${ }^{20}$ and the $16.5 \%$ (any ICH) and $4.7 \%$ in Dargazanli et al and the ETIS Registry investigators' study. ${ }^{21}$ Although there are differences between the above studies, such as patient eligibility and variable criteria, leading to different results, the overall tendency favors endovascular therapy in patients with MIS and LVO.

Moreover, we found no statistical difference in safety between the 2 groups. However, the sICH rate of the group treated in $\leq 6$ hours was higher than that of the group treated in $>6$ hours. Traditionally, extended time from onset to vascular recanalization is associated with higher intracranial hemorrhagic risk. ${ }^{22,23}$ The higher proportion of hypertension history and large vessel occlusion of determined etiology in the group treated in $\leq 6$ hours might contribute to this difference. Another explanation might be that ASPECTSs in the group treated after $>6$ hours were better than those in the group treated in $\leq 6$ hours. These findings indicate that smaller infarct volumes may be favorable. However, further analysis with more samples is needed.

To date, there are still no randomized clinical trials analyzing the effect of endovascular treatment for MIS. Goyal et $\mathrm{al}^{24}$ and the HERMES collaborators conducted a meta-analysis of 5 major mechanical thrombectomy clinical trials. Only 177 patients with MIS were available for analysis, but they concluded that the direction of effect favored endovascular treatment for patients with MIS. The recent DAWN study also showed that patients with stroke treated 6-24 hours from the onset of symptoms also benefited from thrombectomy when they had salvageable brain tissue. ${ }^{12}$ The main finding of our study is that endovascular treatment for patients with MIS 6-24 hours from symptom onset should be determined on the basis of clinical and imaging mismatch assessments.

This was a multicenter study in the Chinese population, but the relatively small sample size and retrospective design limited its power. Further prospective, randomized, controlled trials are warranted.

\section{CONCLUSIONS}

We found that the efficacy and safety of endovascular treatment were not heterogeneous $\leq 6$ and 6-24 hours from symptom onset to groin puncture in patients with anterior circulation MIS and LVO and imaging mismatch based on perfusion CT or diffusionweighted MR imaging.

\section{ACKNOWLEDGMENTS}

The authors sincerely thank all hospitals and participants for their help and thank Shiquan Yang (Department of Neurology, No.123 Hospital of the People's Liberation Army, Bengbu, Anhui, China) for providing cases. We also thank the reviewers for their helpful comments.

Disclosures: Xianjin Shang—RELATED: Grant: This study was partly supported by National Natural Science Foundation of China (No. 81530038, 81400993 and 81671172), National Key Research and Development Program (No.2017YFC1307901), Chinese Postdoctoral Science Fund (No. 2015M572815), and the scientific research fund of Wannan Medical College*. Min Lin—RELATED: Grant: This study was partly supported by National Natural Science Foundation of China (No. 81530038, 81400993 and 81671172), National Key Research and Development Program (No.2017YFC1307901), Chinese Postdoctoral Science Fund (No. 2015M572815), and the scientific research fund of Wannan Medical College.* Shun Li-RELATED: Grant: This study was partly supported by $\mathrm{Na}$ tional Natural Science Foundation of China (No. 81530038, 81400993 and 81671172), National Key Research and Development Program (No.2017YFC1307901), Chinese Postdoctoral Science Fund (No. 2015M572815), and the scientific research fund of Wannan Medical College.* Wenjie Zi_RELATED: Grant: National Natural Science Foundation of China (No. 81530038, 81400993 and 81671172), Comments: This study was partly supported by National Key Research and Development Program (No.2017YFC1307901), Chinese Postdoctoral Science Fund (No. 2015M572815), and the scientific research fund of Wannan Medical College.* *Money paid to the institution.

\section{REFERENCES}

1. Jovin TG, Chamorro A, Cobo E, et al; REVASCAT Trial Investigators. Thrombectomy within $\mathbf{8}$ hours after symptom onset in ischemic stroke. N Engl J Med 2015;372:2296-306 CrossRef Medline

2. Campbell BC, Mitchell PJ, Kleinig TJ, et al; EXTEND-IA Investigators. Endovascular therapy for ischemic stroke with perfusion-imaging selection. $N$ Engl J Med 2015;372:1009-18 CrossRef Medline

3. Saver JL, Goyal M, Bonafe A, et al; SWIFT PRIME Investigators. Stent-retriever thrombectomy after intravenous t-PA vs. t-PA alone in stroke. $N$ Engl J Med 2015;372:2285-95 CrossRef Medline

4. Goyal M, Demchuk AM, Menon BK, et al; ESCAPE Trial Investigators. Randomized assessment of rapid endovascular treatment of ischemic stroke. N Engl J Med 2015;372:1019-30 CrossRef Medline

5. Berkhemer OA, Fransen PS, Beumer D, et al. A randomized trial of intraarterial treatment for acute ischemic stroke. N Engl J Med 2015; 372:11-20 CrossRef Medline

6. Heldner MR, Zubler C, Mattle HP, et al. National Institutes of Health stroke scale score and vessel occlusion in 2152 patients with acute ischemic stroke. Stroke 2013;44:1153-57 CrossRef Medline

7. Nedeltchev K, Schwegler B, Haefeli T, et al. Outcome of stroke with mild or rapidly improving symptoms. Stroke 2007;38:2531-35 CrossRef Medline

8. Rajajee V, Kidwell C, Starkman S, et al. Early MRI and outcomes of untreated patients with mild or improving ischemic stroke. Neurology 2006;67:980-84 CrossRef Medline

9. Smith EE, Abdullah AR, Petkovska I, et al. Poor outcomes in patients who do not receive intravenous tissue plasminogen activator because of mild or improving ischemic stroke. Stroke 2005;36:2497-99 CrossRef Medline

10. Heldner MR, Jung S, Zubler C, et al. Outcome of patients with occlusions of the internal carotid artery or the main stem of the middle cerebral artery with NIHSS score of less than 5: comparison between thrombolysed and non-thrombolysed patients. J Neurol Neurosurg Psychiatry 2015;86:755-60 CrossRef Medline

11. Smith EE, Fonarow GC, Reeves MJ, et al. Outcomes in mild or rap- 
idly improving stroke not treated with intravenous recombinant tissue-type plasminogen activator: findings from Get With The Guidelines-Stroke. Stroke 2011;42:3110-15 CrossRef Medline

12. Nogueira RG, Jadhav AP, Haussen DC, et al; DAWN Trial Investigators. Thrombectomy $\mathbf{6}$ to $\mathbf{2 4}$ hours after stroke with a mismatch between deficit and infarct. N Engl J Med 2018;378:11-21 CrossRef Medline

13. Adams HP Jr, Bendixen BH, Kappelle LJ, et al. Classification of subtype of acute ischemic stroke: definitions for use in a multicenter clinical trial-TOAST. Trial of Org 10172 in Acute Stroke Treatment. Stroke 1993;24:35-41 Medline

14. Higashida RT, Furlan AJ, Roberts H, et al; Technology Assessment Committee of the American Society of Interventional and Therapeutic Neuroradiology, Technology Assessment Committee of the Society of Interventional Radiology. Trial design and reporting standards for intra-arterial cerebral thrombolysis for acute ischemic stroke. Stroke 2003;34:e109-37 CrossRef Medline

15. Tomsick T, Broderick J, Carrozella J, et a; Interventional Management of Stroke II Investigators. Revascularization results in the Interventional Management of Stroke II trial. AJNR Am J Neuroradiol 2008;29:582-87 CrossRef Medline

16. von Kummer R, Broderick JP, Campbell BC, et al. The Heidelberg Bleeding Classification: classification of bleeding events after ischemic stroke and reperfusion therapy. Stroke 2015;46:2981-86 CrossRef Medline

17. Minnerup J, Wersching $\mathrm{H}$, Teuber $\mathrm{A}$, et al; REVASK Investigators. Outcome after thrombectomy and intravenous thrombolysis in patients with acute ischemic stroke: a prospective observational study. Stroke 2016;47:1584-92 CrossRef Medline
18. Mokin M, Masud MW, Dumont TM, et al. Outcomes in patients with acute ischemic stroke from proximal intracranial vessel occlusion and NIHSS score below 8. J Neurointerv Surg 2014;6:413-17 CrossRef Medline

19. Dargazanli C, Consoli A, Gory B, et al; ETIS investigators. Is reperfusion useful in ischaemic stroke patients presenting with a low National Institutes of Health Stroke Scale and a proximal large vessel occlusion of the anterior circulation? Cerebrovasc Dis 2017;43: 305-12 CrossRef Medline

20. Pfaff J, Herweh C, Pham M, et al. Mechanical thrombectomy in patients with acute ischemic stroke and lower NIHSS scores: recanalization rates, periprocedural complications, and clinical outcome. AJNR Am J Neuroradiol 2016;37:2066-71 CrossRef Medline

21. Dargazanli C, Arquizan C, Gory B, et al; ETIS REGISTRY Investigators. Mechanical thrombectomy for minor and mild stroke patients harboring large vessel occlusion in the anterior circulation: a multicenter cohort study. Stroke 2017;48:3274-81 CrossRef Medline

22. Khatri P, Wechsler LR, Broderick JP. Intracranial hemorrhage associated with revascularization therapies. Stroke 2007;38:431-40 CrossRef Medline

23. Hao $\mathrm{Y}$, Yang D, Wang H, et al; ACTUAL Investigators (Endovascular Treatment for Acute Anterior Circulation Ischemic Stroke Registry). Predictors for symptomatic intracranial hemorrhage after endovascular treatment of acute ischemic stroke. Stroke 2017;48: 1203-09 CrossRef Medline

24. Goyal M, Menon BK, van Zwam WH, et al; HERMES collaborators. Endovascular thrombectomy after large-vessel ischaemic stroke: a meta-analysis of individual patient data from five randomised trials. Lancet 2016;387:1723-31 CrossRef Medline 